\title{
Implications of an Interpretive Understanding of LCA Practice
}

\author{
Emma Rex and Henrikke Baumann* \\ Department of Energy and Environment, Division of Environmental Systems Analysis, \\ Chalmers University of Technology, Göteborg, Sweden
}

\begin{abstract}
Despite the desirability of a life cycle perspective and many attempts to facilitate life cycle assessment (LCA), industry has been relatively slow to adopt LCA. In this research, we thus set out to investigate the underpinnings of LCA practice in industry. A literature review shows that the present understanding is that 'structural' conditions such as location and sector determine the use of LCA in industry. However, a field study of two companies in the Swedish forest product industry (thus in the same sector and country) indicates that LCA practice is shaped more by individual preferences and chance events. Our results imply a more interpretive understanding of LCA practice than has been put forward by previous research. The two types of understanding are discussed and their implications for practitioners in industry, organizations promoting the use of LCA and further research are presented. Copyright (C) 2008 John Wiley \& Sons, Ltd and ERP Environment.
\end{abstract}

Received 6 June 2005; revised 28 August 2006; accepted 19 September 2007

Keywords: field study; forest product industry; interpretive explanations; LCA practice; organization theory; Sweden

\section{A Desired Course}

T

HE CONSIDERATION OF ENVIRONMENTAL IMPACTS DURING THE ENTIRE LIFE CYCLE OF THE PRODUCT, FROM 'CRADLE to grave', is increasingly considered by various actors in society as a necessary way of obtaining more sustainable production and consumption patterns. A more holistic approach to environmental improvements enables action to be taken where it is most needed and can avoid environmental sub-optimizations along the product chain. This way of thinking is advocated not only by authorities such as the European Communities (Commission of the European Communities, 2003) but also by companies through their environmental policies (e.g. Volvo, 2004; Nokia, 2006) as well as by the general public (Heiskanen, 2002).

Life cycle assessment (LCA) is a tool that can be used in industry to assess the environmental impact of a product throughout its entire life cycle. It was developed in the late I960s and introduced on a broad scale to industry in the early I990s. It has many areas of application and can affect functional areas in a company such as product development, purchasing and marketing. LCA is, however, an ambitious tool. It requires companies to address activities outside their control and outside their traditional responsibilities. It has repeatedly been criticized for being too complex, time consuming and costly for industrial use. Researchers, companies and various organizations have all attempted to address these problems in order to encourage and facilitate the use of LCA in industry. Nevertheless, its use is still quite limited.

* Correspondence to: Henrikke Baumann, Ph.D., Associate Professor, Department of Energy and Environment, Division of Environmental Systems Analysis, Chalmers University of Technology, 41296 Göteborg, Sweden. E-mail: henrikke.baumann@chalmers.se 


\section{Measures to Facilitate the Use of LCA}

When LCA was introduced in industry, many 'technical problems' of conducting and using the tool came into focus. Researchers set out to address these problems; LCI (life cycle inventory) databases were created to ease data collection, a format for handling the data within these databases was developed and tools and methods were designed to ease calculation procedures (Baumann and Tillman, 2004). It was recognized that there was a need for a uniform 'harmonized' LCA methodology to make it possible to use LCA in areas such as comparative environmental marketing (Baumann and Tillman, 2004). Starting in I993, a whole series of ISO standards for LCA was developed. The first standard (ISO I4040-Life Cycle Assessment: Principles and Frameworks) was published in 1997.

Over the years, much effort has been put into developing tools and methods to reduce the time spent on data collection and calculation in LCA studies. Researchers and practitioners have repeatedly emphasized the need for user-friendly, quality-assured, comparable and consistent LCI databases (see Smet and Stalmans, I996; Sonnemann and Inaba, 2002; Frischknecht et al., 2005). In response, an increasing number of national and regional LCA (or LCI) databases are being developed (Frischknecht, 2006). Examples include a database for energy production in Korea (Lee et al., 2004) and the Swiss ecoinvent database (see Frischknecht et al., 2005).

The large number of data needed has been identified as one of the main obstacles to the use of LCA by small and medium-sized companies (see, e.g., Mueller et al., 2004; Zamagni et al., 2005). One way to address this problem is to develop ways of reducing the number of data needed. A number of simplified LCA methods have thus been developed (Mori et al., 2000; Fleischer et al., 200I; Hochschorner and Finnveden, 2003), and some tools have been developed specifically for small and medium-sized companies (see e.g. Cappellaro et al., 2005; Goldoni and Bonoli, 2005).

However, despite more than a decade of intense efforts to refine the methodology, set standards, develop tools and provide recommendations to facilitate the conduct of an LCA, the adoption of LCA in industry at large is slow. Companies seem to be hesitant to use it. Heiskanen (2002) concluded that many companies are still uncertain, or 'dabbling' with LCA.

What can be done to solve this problem? One approach emphasized in the literature is to continue to develop tools and methods. Sonnemann (200I) recognized the limited use of LCA in industry and identified the lack of consistent information systems for LCI data and of widely recognized LCA methods as the two main obstacles to its adoption. Frischknecht et al. (2005) observed the low penetration of LCA in industry and argued that 'actual realization of life cycle approaches in practice' would require user-friendly access to databases and assured quality of the life cycle data.

However, the question arises of whether we have found and dealt with all the major barriers to the implementation and use of LCA in industry. Work on LCA databases is as old as the LCA concept itself. The problem may be that the introduction of LCA and related concepts in industry represents a new way of thinking about environmental issues and requires a deeper understanding of the premises underlying the life cycle principle and its adoption. To understand and influence the processes at work, we need to study LCA practice in industry to determine how it is shaped, what the driving forces are, and its application and nature.

\section{Understanding Industry Practice}

Most research on LCA has been devoted to developing methodology and finding technical solutions that facilitate LCA studies. This research is based largely on assumptions about what constitute the barriers in industry. A smaller body of research has investigated the actual practice of LCA in industry. A few attempts have been made to identify the rationales companies give for using LCA. The reasons identified can be divided into two main types: external pressure from regulations and internal company motivation (e.g. advantages in competition). Explanations vary as regards the relative importance of these two rationales. None of the seven companies studied by Verschoor and Reijnders (1999) mentioned response to regulations as a reason for implementing life cycle studies. Berkhout and Howes (I997), however, found regulatory and market drivers to be equally common as the primary motivation for adopting LCA. 
Another set of studies maps out the distribution and use of LCA in industry. Typically these studies distribute questionnaires to industry representatives in an attempt to take a 'snapshot' of the current situation. Such surveys tell us something about the existence and use of LCA in industry at a certain moment in time. Thus we know that the environmental, research and product development departments are the departments most engaged in carrying out LCA studies in Denmark (Broberg and Christensen, I999); that the most important application for LCA in Sweden and Germany is bottleneck identification (Frankl and Rubik, 2000) and that the forestry/pulp and paper industry is the industry with the highest share of companies using LCA in Sweden (Baumann, I996). All these studies conclude that industry practice of LCA varies.

The most common way to analyse why company practices vary has been to analyse these types of survey, seeking correlations between the characteristics of companies and their use of LCA. In one such study, Frankl and Rubik (2000) found that industry use of LCA varies with company size and the country in which the company operates. In another, Berkhout and Howes (I997) found 'sector-specific approaches' and concluded that the nature of LCA is determined mainly by the life cycle position of the industry and the nature of competition in that industry.

\section{Inviting Social Science}

Like most areas of industrial ecology, LCA is dominated by researchers with a background in engineering or natural science (Korhonen et al., 2004). It is not surprising that such researchers tend to adopt a rather technocratic approach, focusing on technical solutions or explaining variations in industry practice by searching for correlations between measurable characteristics.

However, for a company to move towards increased life cycle thinking also involves a shift in how environmental problems and responsibilities are defined. New tools such as LCA require companies to investigate activities that have traditionally fallen outside their responsibilities. Attention thus needs to be paid to issues involving organizational change with regard to how environmental issues are dealt with. The literature on organizational change emphasizes, for example, that an understanding of the organizational culture is necessary if change is to be successful (Heracleous, 200I). Culture is a wide concept, including artefacts as well as a sense of how things 'ought to be' and the basic assumptions of the employees (Schein, I984, I992, cited by Heracleous, 200I). However, such issues of organizational culture will not show in most of the 'technocratic' research accounted for above.

A small but increasing number of researchers have adopted a different approach to understanding the use of LCA and see it as part of a social or organizational process. In their editorial in Business Strategy and the Environment, Korhonen et al. (2004) argue that 'actual change' in industrial ecology will not occur unless we understand and influence human behaviour. To achieve this, engineering tools such as LCA must be linked with aspects of the social sciences. An understanding of the broader organizational context seems to be necessary to understand and be able to influence LCA practice in industry.

There are, as yet, very few examples of research on LCA practice inspired by the social sciences. Among the few are the work of Heiskanen (2000), Poikkimäki (2006), Baumann (1998, 2000) and Rex and Baumann (2004). What these studies have in common is a focus on the actors and their actions, role and interpretations as regards LCA work in industry. In this article we argue that this perspective is important for understanding LCA in industry and that it will lead to alternative recommendations for the promotion of LCA. In the following, examples of what this research perspective offers are shown through a study where an 'interpretive-based understanding' became necessary for explaining the varying LCA practice observed in industry (see also Rex and Baumann, 2004).

\section{LCA Practice in the Swedish Forest Product Industry}

In 2003-2004, we studied the practice of LCA in two companies in the Swedish forest product industry: Stora Enso and SCA. The aim of the study was to understand LCA practice and how it was shaped in the two companies. Our investigative strategy for empirically based theorizing was based on the work of Glaser and Strauss (I967).

We conducted exploratory field studies, mapping out LCA studies done by these companies from the introduction of the tool in the late I980s until 2003, taking careful note of their organizational and historical context. Attention 
was focused on the 'core people', that is, those who were part of operating LCA units and who were working daily with LCA. LCA activity was broadly defined to include LCA studies and methodology as well as related actions (such as the introduction of life cycle thinking in environmental management) and the interplay between actors over time. Data were collected from internal company documents and external company reports. Interviews were conducted with former and current employees at each company who had played a key role in LCA work (four informants for each company). In all, the analysis was based on 78 documents from Stora Enso and I34 documents from SCA. These documents provided information on topics such as LCA studies, the organization of LCA work and external and internal communication.

Given earlier research findings and the similarities between the companies studied (both operating in the same sector, both in Sweden, and both with more than ten years' experience with LCA) we expected to find relatively similar LCA practices. However, the main finding of our study was that there were considerable differences in LCA practice between the two companies (Rex and Baumann, 2004, 2007). These differences are examined in this paper and lead into a discussion of different approaches to research and the understanding of LCA practice, and of the practical implications of shifting the dominant understanding of LCA practice in industry.

\section{Diverging LCA Practices}

There were striking dissimilarities in the LCA work in the two companies in areas ranging from the type and number of LCA studies to how LCA work was organized and approached. These dissimilarities are summarized in Table I and are then briefly described in what follows. More detailed information can be found in the work of Rex and Baumann (2004, 2007).

\section{Differences in Number of Studies, Methodology and Organization}

The most striking difference between the companies was the number of LCA studies conducted and the settings of these studies. During the studied period, SCA carried out seven times as many studies as Stora Enso (94 compared with I3 studies). SCA also had a higher proportion of its studies done in-house (94\% in house and 6\% in collaboration with other companies or organizations), while Stora Enso conducted a larger proportion of its studies in collaboration with other industries and organizations (60\% in house and $40 \%$ as joint studies).

The large difference in the number and types of LCAs can be ascribed to the fact that LCA became part of the formal product development process at SCA. An LCA study was compulsory whenever certain types of product were being developed. These 'routine LCAs', which involved comparison of old and new versions of the same product, accounted for $83 \%$ of all the studies. No such 'routine LCA' existed at Stora Enso. Their studies were conducted in a number of contexts and ranged from stand-alone studies to comparisons of processes and comparisons of different products.

Not only did the number and type of LCA studies differ, but so also did the approach to methodological issues. For example, Stora Enso restricted the use of weighting, while SCA developed their own weighting method.

\begin{tabular}{lll}
\hline & Stora Enso & SCA \\
\hline No. of studies (1993-2003) & 13 & 94 \\
No. of people working daily with LCA & $1-2$ & $2-4$ \\
Proportion of studies done in-house & $60 \%$ & $94 \%$ \\
Type of study & Varied & $\begin{array}{c}\text { Mainly comparison of old and new versions } \\
\text { of a product }\end{array}$ \\
Organization of LCA work & Spread/fragmented throughout the & Mainly in one department \\
& organization &
\end{tabular}

Table 1. Examples of diverging LCA practice in the two companies 
Moreover, while the vast majority of LCAs at SCA were cradle to grave, the preferred scope at Stora Enso shifted with time to cradle to gate.

The organization of the LCA work also differed markedly between the two companies, although in both the group of LCA practitioners was small and informally organized. Most LCA work at SCA originated in the environmental department of a particular product group. By contrast, LCA work at Stora Enso was more widespread throughout the organization. LCA core people worked in a support function at the corporate level and LCA studies were conducted at various paper mills.

\section{Reasons for Differing Practice}

Why were there so many differences in LCA practice in the two companies? Since we had chosen to study companies with similar sector affiliations, sizes, locations and experiences with LCA, the variations we found were surprisingly large. Also, we could not use these parameters to explain the differences found. Seeking to explain the differences, we studied LCA practice in these companies over time and followed peoples' actions and rationales. We found that the differing practices could be explained by the way people made sense of various situations and by the historical and organizational context of each organization.

\section{Choice of Products to Assess}

In both companies, products were not chosen for assessment on the basis of some conscious strategic decision by top management. Rather, the choice seemed to be largely determined by who became interested in LCA as a tool and their strategies for developing this interest.

At Stora Enso, LCA work was long driven by a single practitioner who kept up the LCA work and extended his own knowledge by engaging in collaborative studies with trade associations, customers and suppliers. When an extra LCA practitioner was hired by the company in the mid-I990s, the interest in external projects faded and more internal studies were undertaken. Studies were done where there was an interest from the mills. At SCA, LCA was introduced by an environmental manager who had previously been involved in researching and developing nappies for babies. A baby nappy was also the first product subjected to LCA studies at SCA. This product group has retained a dominant position in the company's LCA work, and LCA procedures within this product group have gradually spread to related products.

\section{Motivation for Continuous LCA Work}

Many of the differences between the two companies' LCA practices derive from the fact that LCA is part of the product development routine at SCA. This formalization of LCA was preceded by years of lobbying on the part of the core LCA people. These people also took advantage of change occasions to get acceptance for LCA. Through continuously renewing part-time projects involving LCA, the environmental manager who was interested in LCA at SCA succeeded in having one process developer work with LCA for several years in order to learn about it and develop internal tools. Moreover, to increase awareness and knowledge of LCA in the industry (and to facilitate data collection for the company's own LCA studies), the environmental manager initiated a 'learning project' in which members of the trade association conducted a joint LCA study.

However, an external coincidence was probably even more important than the broader recognition of LCA within the industry. Eco-labelling of nappies became a hot issue in the mid-I99os, and there were many questions from consumers about why SCA did not label its products. The company had several reasons for not using labels, including the fact that existing labels restricted product design and might actually inhibit environmental improvements. However, acting on behalf of a private label agent, SCA manufactured an eco-labelled nappy. An LCA of this product revealed that environmentally it was no better than SCA's own unlabelled ones. This LCA provided useful arguments that could be used to explain why SCA chose not to use a label. Moreover, it provided SCA with a hands-on example of the usefulness of LCAs. 
The unwanted eco-label thus gave unexpected help to the core LCA people, who took advantage of the situation to promote continued engagement with LCA at SCA. This increased company acceptance of LCA and awareness of its perceived benefits. Ordering an LCA was accepted as a formal part of the product development process about a year after the LCA of the labelled nappy. From then on, the number of LCA studies at SCA increased markedly, and new people were hired to help carry them out.

\section{Methodological Considerations}

Our field study showed that peoples' interests and actions seem to influence not only the organization of the LCA work but also the LCA methodology used. One example concerns the weighting of different environmental impacts. The issue of weighting was much discussed in the LCA community in the mid-I99os (see the work of Baumann and Tillman, 2004, for an overview). Several weighting systems based on different values and world-views were developed. However, neither Stora Enso nor SCA felt confident about using any of the existing weighting methods.

The LCA practitioners at Stora Enso were accustomed to seeking guidance on methodological issues through internal and external discussion. For example, they conducted 'LCA seminars' involving participants from different parts of the company as well as external experts. Many people in house, including the newly hired LCA practitioner, thought the existing weighting systems would give rise to unfair comparisons and be disadvantageous to the paper industry. Consequently, the practitioners limited their use of weighting methods and actively supported further research on LCA.

SCA handled the problem of weighting differently. Most of their LCA tools had been developed in-house by a single person. Methodological issues were generally not discussed at length and were typically decided on within the small group of practitioners. Instead of discussing whether to use weighting methods and, if so, which external method was the most consistent with the values of SCA, the LCA practitioner at SCA simply designed an independent weighting method based on the priorities stated in SCA's environmental policy.

\section{Differing Approaches}

The examples given above reflect how the interests and actions of individuals and their interaction with people within and outside the organization had a decisive impact on the shaping of LCA practice. The LCA practitioners acted according to how they perceived the situation and how they had previously dealt with LCA. Over time, two different approaches to LCA emerged within the companies: one could be called 'pragmatic' and the other 'scientific'. According to the pragmatic view at SCA, LCA is practical, internal and operational. The use of LCA is now a compulsory and integrated part of the product development process for certain product groups. By contrast, the people at Stora Enso had a more scientific approach to LCA and regarded it as a tool in need of further development. The attitude of the LCA core people at Stora Enso was thus restrictive, characterized by discussions about the reliability of data and the 'scientificness' of the method.

Individuals' actions and the organizational experience of LCA not only influenced certain decisions but also had enduring effects on how LCA came to be seen throughout the organization. The actions taken both shaped and were shaped by the organizational understanding of the tool and its application. This process can be compared with Weick's (I995) concept of 'sense-making' in organizations. Weick suggests that sense-making is an ongoing process that decides how we interpret and explain actions. It is also the process of selecting and generating what is being interpreted. Hence, people both interpret a situation and construct the cues that are interpreted. The two approaches to LCA can thus be said to reflect different ways of making sense of the use of LCA.

\section{Functional Versus Interpretive Explanations}

Our field studies showed that the studied companies differed considerably and fundamentally in their LCA practices. This finding in itself is nothing new. Different LCA practices have been observed in several studies prior to ours, as outlined in the introduction. The difference between our studies and the other studies lies in where the 
differences in LCA practice were sought and how they were explained. Earlier explanations of differences in LCA practice were based on structural characteristics of the companies, such as their sector affiliation, location or size. However, such structural characteristics could not be used to explain the results obtained in our study and we had to seek other explanations. Our analysis suggests that the shaping of LCA work is driven by particular individuals. Their experience, preferences and actions affected what was studied and how it was studied, and resulted in a certain logic in the approach to LCA within each company.

Explaining divergent LCA practices on the basis of individuals' sense-making is fundamentally different from explaining them on the basis of structural characteristics of the company. The two explanations reflect different assumptions about how we understand the way in which 'reality' is constituted. The philosophy of science identifies several perspectives on reality, two of which are important here, namely whether reality is considered to be 'objectively determined' or 'socially constructed'. Drawing on terminology used by researchers who focus on organizational studies such as Burrell and Morgan (I979) and Czarniawska-Joerges (I992), we refer to the explanations based on these perspectives as functionalist and interpretive, respectively.

According to the functionalist perspective, people respond to external stimuli in a deterministic way. From the interpretive perspective, people's actions are the results of how they make sense of a situation. Czarniawska-Joerges provides a useful image of how the two schools explain the relation between people and an organization: in the functionalist approach, people are in organizations for the sake of the survival of the organization; in the interpretive approach, people are not only in organizations but also create them. In the literature, other terms used to denote a perspective in which reality is considered to be objectively determined include structuralist (Bauhn, 2004) and positivist (Easterby-Smith et al., I99I). Similarly, the socially constructed perspective can be referred to as phenomenological (Easterby-Smith et al., I99I) and constructivist (Morcöl, 200I).

\section{The Functionalist Approach}

In the functionalist approach, the social world is assumed to be made up of artefacts and relationships, both of which can be identified and measured (Burrell and Morgan, 1979). By scrutinizing the intercorrelations between various characteristics of the organization and its surroundings, it is possible to find laws and regularities that can be used to explain the behaviour of the organization. The ideas and intentions of individuals are not decisive for what happens (Andersen and Kaspersen, 2003). People do act, but the reasons why they act in a certain way are external to the individual: people respond in a deterministic way to external stimuli (Easterby-Smith et al., I99I). Researchers in this tradition seek causal regularities external to the individual to explain the behaviour of the system. Such explanations may be structural, and when applied to the practice of LCA in industry they suggest that companies with similar structural characteristics (such as belonging to the same industry sector, being the same size or operating in the same country) will use LCA in a similar way.

\section{The Interpretive Approach}

In the interpretive approach, the point of view of actors is regarded as important for understanding social affairs (Burrell and Morgan, I979). Reality is considered to be 'socially constructed' and each individual acts according to how he or she makes sense of the situation (Easterby-Smith et al., I99I). Researchers within the interpretive tradition reflect the view that 'society' is constituted and maintained and seek to comprehend it by understanding the sense-making of the people involved. Applied to the practice of LCA in industry, this school of thought does not perceive the shaping of LCA as primarily dependent on external factors but on how people perceive and act in various situations. Individual actions are thought to have considerable influence on how LCA work is carried out, and the behaviour of individuals sets the scene for further perceptions and actions.

\section{The Most Common Research Perspective}

Both the functionalist and the interpretive approach are used in research on organizations (Jackson, I993). However, the functionalist approach has been the dominant framework for the academic study of organizations 
in the 2oth century and has produced the largest share of the theory and research within organization studies (Burrell and Morgan, I979). Within the LCA community, a functionalist approach has also dominated thinking about LCA practices for many years, as shown by studies that seek to explain variations in LCA practice by examining company variables such as size or industry affiliation. The repeated attempts to facilitate data collection and LCA calculations also seem to assume a functionalist understanding of LCA practice, for they focus on technical matters and external premises in order to promote LCA use in industry.

How LCA practice is understood has a decisive impact on what actions are recommended to discourage companies from merely 'dabbling' in LCA. According to the functionalist view, the best way of working with LCA in one company will also be the best for other companies with similar structures. Thus it is frequently assumed that sector affiliation is a major determinant of LCA practice, and thus sector-wise recommendations have been put forward to policy-makers (e.g. SPRU, I996) and standardization bodies (e.g. Weidema, I996). A sectorial orientation has also been the starting point for collaboration on LCI databases and methods (see, e.g., Svending, 200I) and for discussions among researchers about how to make LCA more practicable (Swiss LCA Forum, 2004). The same is true of many initiatives that aim to promote LCA use in industry. One ongoing example is the UNEP/ SETAC Life Cycle Initiative, launched in 2002, where one of the objectives is to 'identify best practice in different industry sectors' (UNEP, 2004).

\section{The Practitioners' View}

The way in which practices in organizations are understood not only affects the work and recommendations of researchers but also the way practitioners act. Brunsson (I985) discusses the implications of people having different 'attributions', and distinguishes between those who attribute situations to the environment and those who attribute them to personal action. This distinction is similar to that between a functionalist or interpretive worldview. According to Brunsson, people who attribute situations to the environment believe that external events are decisive for what will happen. Such people emphasize predicting and adapting to the future. People who attribute situations to their own actions have a stronger sense of personal control and see people's actions as decisive for what will happen. Their focus will be on planning for the future and on generating commitment and motivation.

A functionalist world-view (environmental attribution) seems to be the most common not only among researchers in the field of organizational studies but also in society, as shown by research such as the study of policy professionals by Morcöl (200I). Our field study of LCA practice in the Swedish forest product industry revealed both kinds of world-view among the LCA practitioners. Some were very conscious of their role in creating the conditions for their work. Thus one practitioner initiated an LCA project in the trade association to increase awareness and facilitate her own data collection.

Other practitioners were more concerned about what they 'ought to do' and tended to turn to external bodies and seek more research for guidance on the right way to carry out their work.

It has been argued that functionalist world-views tend to decrease the perceived responsibility and scope of action of individuals. Bauhn (2004), for example, points out that functionalist assumptions such as 'I am a woman, hence I am discriminated against' limit the scope for working for greater social equality. Welford (I998) argues that people's realization that they are 'capable of building their own future structures' is of great importance for any change process within industry. Hence, it could be argued that a more interpretive approach would probably change the premises for initiatives advanced by LCA practitioners. If the 'best practice' is not determined by external causes, then it has to be acknowledged that people create the organization and that people thus have to work actively to construct and shape LCA work. The realization that LCA practice is shaped by individuals and not determined by external conditions means that it is not only possible but also necessary for people within companies to work consciously and actively to create strategies for application and implementation of LCA in each organization and situation. In this process, inspiration for ways of working with and benefiting from LCA need not be limited to the current trend in their own industry, but can be sought across industries. 


\section{Implications of an Interpretive Understanding for Researchers and Practitioners}

Many policy-makers and industry representatives want to see life cycle considerations taken into account in industrial society. However, the many attempts to facilitate use of LCA seem to have had limited impact on the adoption of LCA in industry as a whole. We argue that one reason for this lack of impact is the failure to grasp the individual and organizational context of LCA work, an aspect that tends to get lost in well intentioned but technocratic attempts to help industry adopt a life cycle perspective.

There is a need for an interpretive understanding of LCA practice. A one-sided focus on structural explanations can become a barrier to the spread of LCA since such explanations allow companies, policy-makers and other actors to narrow down their perceptions of possible or 'suitable' LCA activities to what has been recommended as best practice for that specific industry. Industry representatives who do not feel comfortable with a particular LCA practice recommended for their sector may then choose not to engage in LCA if recommendations lack sense to them.

A change from the dominant structural understanding to an interpretive perspective will result in a new set of recommendations to people who want to promote LCA. An interpretive view of LCA practice will allow greater scope of action for people working with LCA in industry. It will encourage them to adapt LCA practices to the specific problems and strategies of the company in which they work, and will provide opportunities for more aware and apt uses of LCA.

Organizations and policy-makers aiming to boost LCA use should thus consider alternatives to simply offering more refined tools and drafting more sector-wide recommendations on how to carry out LCA work. They might find that an exhibition of various uses and practices of LCA may be a more efficient means of encouraging LCA use. Measures could also be taken to encourage and strengthen LCA practitioners within the companies. One organization that has adopted such an approach is the Swedish Centre for Environmental Assessment of Product and Material Systems (www.cpm.chalmers.se). Within the network associated with this centre, people working with LCA in different industries share their experience and receive general coaching in such areas as communication, motivation and interaction.

In line with the recommendations above, we encourage those researching LCA practice to study and present various ways of using and benefiting from LCA work. We also welcome sociological, organizational and environmental management research that will complement the technical perspective in LCA practice.

\section{Conclusions}

Studying LCA practice in two similar companies revealed considerable differences in the scope and nature of their LCA activities and in their approaches to LCA. These differences could not, as is often claimed, be explained by structural factors such as the size of the companies, their sector affiliation or location. Instead, we found that the actions and sense-making of individuals played an important role in shaping each company's LCA work. These results suggest a new way of understanding what shapes LCA practice. A shift towards such interpretive understanding will mean a new set of recommendations for the promotion of LCA in industry and calls for further study of LCA practices in an individual and organizational context. An interpretive approach also changes the premises for LCA practitioners, assigning them the opportunity and the responsibility to work consciously and actively to promote LCA. With this approach, we hope much industry LCA practice will evolve beyond mere 'dabbling'.

\section{Acknowledgements}

This research would not have been possible without the kind support of Stora Enso and SCA. We are also grateful to the Swedish Environmental Protection Agency for funding the research program FLIPP (Furthering Life Cycle Considerations Through Integrated Product Policy), within which this research has been carried out. 


\section{References}

Andersen H, Kaspersen LB (eds). 2003. Klassisk och Modern Samhällsteori. Studentlitteratur: Lund, Sweden.

Bauhn P. 2004. Vi har gått vilse bland strukturerna [We have got lost among the structures]. Axess 2004(5).

Baumann H. I996. LCA use in Swedish industry. International Journal of LCA I(3): I22-I26.

Baumann H. I998. Life Cycle Assessment and Decision Making: Theories and Practices, PhD thesis. Department of Technical Environmental Planning, Chalmers University of Technology: Göteborg, Sweden.

Baumann H. 2000. Introduction and organisation of LCA activities in industry. International Journal of LCA 5(6): 363-368.

Baumann H, Tillman A-M. 2004. The Hitch Hiker's Guide to LCA. Studentlitteratur: Lund, Sweden.

Berkhout F, Howes R. I997. The adoption of life-cycle approaches by industry: patterns and impacts. Resources, Conservation and Recycling 20(2): 7I-94.

Broberg O, Christensen P. I999. LCA experiences in Danish industry. International Journal of LCA 4(5): $257-262$.

Brunsson N. I985. The Irrational Organisation: Irrationality as a Basis for Organizational Action and Change. Wiley: Chichester.

Burrell G, Morgan G. I979. Sociological Paradigms and Organisational Analysis. Heinemann: London.

Cappellaro F, Bertuzzi D, Naldesi L. 2005. On-line green tools and services for small and medium sized enterprises. 12th SETAC Europe LCA Case Studies Symposium, Bologna, 2005.

Commission of the European Communities. 2003. Communication from the Commission to the Council and the European Parliament, Integrated Product Policy. Brussels.

Czarniawska-Joerges B. I992. Exploring Complex Organizations: a Cultural Perspective. Sage: Newbury Park, CA.

Easterby-Smith M, Thorpe R, Lowe A. I99I. Management Research. Redwood: Trowbridge, UK.

Fleischer G, Gerner K, Kunst H, Lichtenvort K, Rebitzer G. 200I. A semi-quantitative method for the impact assessment of emissions within a simplified life cycle assessment. International Journal of LCA 6(3): I49-I56.

Frankl P, Rubik F (eds). 2000. Life Cycle Assessment in Industry and Business. Springer: Heidelberg.

Frischknecht R. 2006. Notions on the design and use of an ideal regional or global LCA database. LCA Special I: 40-48.

Frischknecht R, Jungbluth N, Althaus H-J, Doka G, Dones R et al. 2005. The ecoinvent database: overview and methodological framework. International Journal of LCA го(3): 3-9.

Glaser BG, Strauss AL. I967. The Discovery of Grounded Theory. Weidenfeld and Nicolson: London.

Goldoni S, Bonoli A. 2005. LCA simplified tools for SMEs. Comparisons between the software VerdEE and the software eVerdEE through the case study of a brick-works. 12th SETAC Europe LCA Case Studies Symposium, Bologna, 2005.

Heiskanen E. 2000. Translations of an Environmental Technique, PhD thesis. Helsinki School of Economics and Business Administration: Helsinki.

Heiskanen E. 2002. The institutional logic of life cycle thinking. Journal of Cleaner Production Io(5): 427-437.

Heracleous L. 200I. An ethnographic study of culture in the context of organizational change. The Journal of Applied Behavioral Science 37(4): $426-446$.

Hochschorner E, Finnveden G. 2003. Evaluation of two simplified life cycle assessment methods. International Journal of LCA 8(3): II9-I28.

Jackson MC. I993. Systems Methodology for the Management Sciences. Plenum: New York.

Korhonen J, von Malmborg F, Strachan PA, Ehrenfeld JR. 2004. Editorial management and policy aspects of industrial ecology: an emerging research agenda. Business Strategy and the Environment 13: 289-305.

Lee K-M, Lee S-Y, Hur T. 2004. Life cycle inventory analysis for electricity in Korea. Energy 29: 87-IOI.

Morcöl G. 200I. Positivist beliefs among policy professionals: an empirical investigation. Policy Sciences 34(3/4): $38 \mathrm{I}-40 \mathrm{OI}$.

Mori Y, Huppes G, Udo de Haes H, Otoma S. 2000. Component manufacturing analysis. International Journal of LCA 5(6): 327-334.

Mueller KG, Lamperth MU, Kimura F. 2004. Parameterised inventories for life cycle assessment: systematically relating design parameters to the life cycle inventory. International Journal of LCA 9(4): 227-235.

Nokia. 2006. Our Environmental Policy. http://www.nokia.com/A4I970I7 [November 2007].

Poikkimäki S. 2006. Look Closer to See Further: Exploring Environmental Life Cycle Management, LCM, PhD thesis. Jyväskylä Studies in Business and Economics, University of Jyväskylä: Jyväskylä, Finland.

Rex E, Baumann H. 2004. Expanding the Green Practice of LCA: the First Decade of Life Cycle Assessment Activity in the Swedish Forest Products Industry, CPM report 2004:I. Göteborg, Sweden.

Rex E, Baumann H. 2007. Individual adaptation of industry LCA practice: results from two case studies in the Swedish forest products industry. International Journal of LCA 12(4): 266-27I.

Smet B, Stalmans M. I996. LCI data and data quality: thoughts and considerations. International Journal of LCA 2: 96-IO4.

Sonnemann G. 200I. The development of best practice in Life Cycle Assessment (LCA): a step forward towards a life-cycle economy. P\&C unit-UNEP DTIE Information Bulletin I(I): 6.

Sonnemann G, Inaba A. 2002. Best practice on LCA: first workshop of the UNEP/SETAC Life Cycle Initiative. ECP Newsletter I9.

SPRU. I996. European Commission: Adoption by Industry of Life Cycle Approaches (LCA): its Implications for Industry Competitiveness and Trade. SPRU-Atlantic Consulting, University of Sussex.

Svending O. 200I. A State-of-the-Art-Study of the Environmental Information Supplied to the Actors of the Swedish Pulp and Paper Industry and the Tools Used to Provide It, CPM Report 2001:6. Göteborg, Sweden.

Swiss LCA Forum. 2004. Swiss LCA Forum 2005 Program Questionnaire, questionnaire sent to customize the forthcoming LCA Discussion Forums 2005 to the interests of the participants. 
UNEP. 2004. The Initiative's Objectives. www.uneptie.org/pc/sustain/lcinitiative/objectives.htm [January 2005].

Verschoor AH, Reijnders L. I999. The use of life cycle methods by seven major companies. Journal of Cleaner Production 7(5): 375-382.

Volvo. 2004. Environmental Policy. www.volvo.com [November 2007].

Weick KE. I995. Sensemaking in Organizations. Sage: Thousand Oaks, CA, US.

Weidema BP. I996. Improving the performance of LCA. 2nd International Conference on EcoBalance, Tsukuba, I996.

Welford RJ. I998. Corporate environmental management, technology and sustainable development: postmodern perspectives and the need for a critical research agenda. Business Strategy and the Environment 7: I-I2.

Zamagni A, Buttol P, Scalbi S, Sára B. 2005. eVerdEE: a web-based LCA tool for SMEs. Description and case study presentation. 12th SETAC Europe LCA Case Studies Symposium, Bologna, Italy, 2005. 